\title{
Physiological and subjective validation of a novel stress procedure: The Simple Singing Stress Procedure
}

\author{
Jenny T. Le ${ }^{1} \cdot$ Poppy Watson $^{1} \cdot$ Denovan Begg $^{1} \cdot$ Lucy Albertella $^{2} \cdot$ Mike E. Le Pelley $^{1}$
}

Accepted: 19 October 2020 / Published online: 17 November 2020

(C) The Psychonomic Society, Inc. 2020

\begin{abstract}
Laboratory stress-induction procedures have been critical in illuminating the effects of stress on human health, cognition, and functioning. Here, we present a novel stress induction procedure, the Simple Singing Stress Procedure (SSSP), that overcomes some of the practical challenges and conceptual limitations of existing procedures in measuring the causal influence of stress on psychological variables. In the stress condition of the SSSP, participants were instructed to sing a song in front of the experimenter while being video- and audio-recorded. Participants were also informed that they would have to sing again at the end of the experiment, and that this second performance would later be assessed by a panel of experimenters. Participants in a no-stress condition instead read lyrics in each phase. Our findings revealed that participants in the stress condition showed significantly higher blood pressure immediately following the initial singing session, as well as heightened salivary cortisol at a latency consistent with the initial singing session, than those in the no-stress condition. Our stress procedure also generated elevations in self-reported stress ratings immediately after the first singing session and subsequently in anticipation of the second singing session, relative to the no-stress condition. Collectively, these findings suggest that the SSSP is a simple and effective stress induction procedure that may be a promising alternative to existing protocols.
\end{abstract}

Keywords Stress induction $\cdot$ Cortisol $\cdot$ Singing $\cdot$ Trier social stress test $\cdot$ Cold pressor

Stress is a pervasive phenomenon that confronts us in almost every domain of life. We all experience varying amounts of stress day-to-day and moment-to-moment, and these variations can influence the foundations of our biopsychological functioning, such as memory (Cornelisse, van Stegeren, \& Joëls, 2011; Kuhlmann, Piel, \& Wolf, 2005; Lupien et al. 2005; Payne et al. 2006; Wolf, 2009), attention (Esterman et al. 2013; Sänger et al. 2014), and decision making (Mather \& Lighthall, 2012; Starcke \& Brand, 2012). Identified as one of the most significant health problems of the 21st century (Kalia, 2002), prolonged or extreme stress has been shown to exert deleterious effects on cognitive development (Kim et al. 2013; King \& Laplante, 2005; Noble et al., 2015), mental health (Bryant, 2011; Caspi et al. 2003; Estryn-Behar et al. 1990), and resistance to drug relapse (Mantsch, Baker, Funk, Le, \& Shaham, 2016).

Jenny T. Le

jenny.le@unsw.edu.au

1 School of Psychology, UNSW Sydney, Sydney, NSW 2052, Australia

2 Turner Institute for Brain and Mental Health and School of Psychological Sciences, Monash University, Melbourne, Australia
Given its influence across a wide range of aspects of psychological functioning, it is no wonder that stress has been the subject of intense investigation for decades (Schwabe \& Schächinger, 2018).

Procedures for inducing stress in laboratory settings have been critical in providing a foundation for studies investigating the impacts of stress on health and cognition. The challenge for laboratory stress-induction procedures has been finding the balance between generating considerable amounts of stress in an efficient way, while staying within the bounds of ethical standards. A range of protocols have been developed with this balance in mind, including procedures in which participants view distressing video clips (Eisenberg et al. 1988), anticipate an electric shock (Bloom \& Trautt, 1977), or recall personal and emotionally arousing events (Ekman et al., 1983). By far the most commonly used laboratory protocol, however, is the Trier Social Stress Test (TSST; Kirschbaum, Pirke, \& Hellhammer, 1993). In the standard TSST, participants are told they have $10 \mathrm{~min}$ to prepare a speech on a given topic, to be delivered to a "selection committee" of three confederates. Following this anticipation phase, participants deliver the speech in front of the selection committee while being video- and audio-recorded. Finally, participants perform a challenging mental arithmetic task in front of 
the committee. As a whole, the TSST incorporates three key conditions that are thought to elicit a considerable stress response - uncontrollability, motivated performance, and social evaluative threat (Dickerson \& Kemeny, 2004; Kirschbaum, Pirke, \& Hellhammer, 1993).

The TSST is often considered the 'gold standard' stress procedure, particularly due to its incorporation of social evaluative threat. This factor has been established as the critical element in laboratory stressors responsible for elevations in the hypothalamic-pituitary-adrenal (HPA) axis (Dickerson \& Kemeny, 2004). Activation of the HPA axis is widely considered to be the key defining factor of stress experience, and hence the marker of a valid stress induction procedure (Allen, Kennedy, Cryan, Dinan, \& Clarke, 2014; Schwabe, Haddad, \& Schachinger, 2008). Consistent with this idea, the TSST has been shown to reliably induce elevations in downstream neuroendocrine responses that reflect activation of the HPA axis, such as adrenocorticotropic hormone (ACTH) and cortisol (Goodman, Janson, \& Wolf, 2017; Kirschbaum et al., 1993). The TSST has also been shown to influence systolic and diastolic blood pressure (Andrews \& Pruessner, 2013; Childs, Dlugos, \& De Wit, 2010; Jezova, Makatsori, Duncko, Moncek \& Jakubek, 2004) and heart rate (Kirschbaum et al., 1993; von Dawans, Kirschbaum, \& Heinrichs, 2011) suggesting that it induces increased activity of the sympathetic nervous system (for an in-depth review of the biological markers of stress from the TSST, see Allen et al. 2014).

Despite being a well-studied and validated paradigm, the TSST has some notable drawbacks as a laboratory stressinduction procedure. First, researchers have noted that the TSST is a complicated procedure to implement (Brouwer \& Hogervorst, 2014; Smeets et al. 2012). The multiple elements of deception and involvement of multiple confederates result in a procedure that can be, in practice, difficult to coordinate. Moreover, relative to other procedures (like the Cold Pressor Test, discussed below), the TSST can take much longer to administer, with most implementations taking between 15 and $20 \mathrm{~min}$ (see review by Allen et al. 2017). It has also been argued that elevations in sympathetic nervous system responses may be, at least in part, due to participants moving between rooms and standing up while delivering the speech (Brouwer \& Hogervorst, 2014), rather than reflecting a more specific influence of the stressful nature of the task itself.

Given the challenges of implementing the TSST, many researchers have instead used the Cold Pressor Test (CPT; first described by Hines \& Brown, 1932). This protocol attempts to induce stress by having participants submerge one hand in an icecold water bath for up to $3 \mathrm{~min}$. Like the TSST, the CPT has been shown to induce elevations in sympathetic arousal, measured by heart rate (al'Absi, Petersen, \& Wittmers, 2002) and skin conductance (Buchanan, Tranel, \& Adolphs, 2006). However, one critical limitation of the CPT is that it does not incorporate social evaluative stress, which (as noted earlier) is the key factor associated with activation of the HPA axis. In line with this limitation, findings of studies assessing the influence of the CPT on HPA axis activation are somewhat mixed: some studies have found that the CPT does not elicit increases in salivary cortisol (Duncko et al., 2007; McRae et al. 2006); other studies have reported small increases (al'Absi, et al. 2002; Gluck et al., 2004); and yet other studies have found evidence of a moderate cortisol response (e.g., McCullough et al. 2015; Schoofs, Wolf, \& Smeets, 2009; Shields et al. 2019), though still notably smaller than that elicited by the TSST (see Shields et al. 2017, for a metaanalysis in the context of studies examining the effect of stress on memory).

Due to the lack of a social-evaluative component, the CPT may be relatively ineffective in activating the HPA axis - a criterion that is often considered imperative to a valid stress induction procedure (Schwabe, Haddad, \& Schachinger, 2008). Moreover, studies which measure subjective responses to the CPT often only record ratings of pain, rather than stress per se (e.g., Bullinger et al. 1984; Geisser, Robinson, \& Pickren, 1992; Kowalczyk, Evans, Bisaga, Sullivan, \& Comer, 2006), and studies which do explicitly measure subjective ratings of stress show only modest increases following the CPT (e.g., Brady et al. 2006; McRae et al. 2006). That is, while the CPT may inherently be painful (and hence result in activation of the sympathetic nervous system), it may not always be subjectively stressful.

This potential limitation of the CPT motivated the development of the Socially-Evaluated Cold Pressor Test (SECPT: Schwabe, Haddad, \& Schachinger, 2008). In this protocol, participants experience the physiological challenge of submerging one hand in an ice-cold water bath while being video-recorded; participants are told that these video recordings are to be later analysed for facial expressions of pain. With the addition of this social-evaluative component, the SECPT has been found to elicit greater increases in salivary cortisol than the standard CPT (Schwabe, Haddad, \& Schachinger, 2008; Smeets et al. 2012), consistent with the idea that social-evaluative threat is particularly important in eliciting activation of the HPA axis (Dickerson \& Kemeny, 2004). A recent meta-analytic review of studies using the SECPT has found that the procedure produces reliable elevations in subjective ratings of stress and autonomic arousal (measured by systolic and diastolic blood pressure: Schwabe \& Schächinger, 2018). In addition to its conceptual advantages over the standard CPT, the SECPT can be quickly administered (3 $\mathrm{min}$ ) by a single experimenter, unlike the TSST. Despite these advantages, it should be noted that both the CPT and the SECPT have a non-zero cost; experimenters must purchase either the apparatus used to cool the water, or the ice, and clean towels to dry the participant's arm after testing. If ice is used, this must be stored and obtained prior to every study, and disposed of following each testing session, adding to experimenter time for preparation. 
In summary, commonly used stress induction protocols (the TSST and CPT) have important practical and/or conceptual limitations, providing an impetus to develop alternative procedures that overcome these issues. Efforts in this direction have highlighted the potential use of singing as a method for eliciting potent stress responses. Singing in front of an audience is, at face value, highly stressful and socially evaluative in nature; this has been used to advantage in previous studies of stress. For example, Harris (2001) had participants sing the American national anthem while being video- and audio-recorded. When each participant viewed the recording of their performance with two experimenters present, participants showed significant elevations in heart rate and blood pressure. In another notable study by Brouwer and Hogervorst (2014), participants were instructed that they would have to sing a song aloud in front of the experimenter and a confederate after a 60-s timer had elapsed. Significant increases in heart rate and skin conductance were found to coincide with the onset of the instruction about the upcoming requirement to sing, remaining high throughout the 60-s anticipation phase and the performance itself (both also remained high even in the few minutes following the performance). More recently, ReschkeHernández et al. (2017) used a singing-based procedure modelled on the TSST to induce stress. Specifically, participants in this study were given $3 \mathrm{~min}$ to prepare for a singing performance. Participants were then walked by the experimenter into another room where two confederate 'expert' vocal judges (typically one male and one female) evaluated each participant's singing performance. Following the singing performance, participants performed a mental arithmetic task aloud for five minutes. During both tasks, participants were video- and audio-recorded, and the confederate judges wrote notes on each participant's performance. Participants were then walked back to the original room to complete subjective measures. This procedure elicited increases in subjective ratings of stress at levels comparable to the TSST. Together, these findings suggest that singing can effectively elicit the physiological and subjective responses that reflect the experience of stress. In relation to the commonly used stress protocols discussed above (the TSST and CPT), the use of singing does not necessitate potentially confounding physical activity, unlike the TSST (Brouwer \& Hogervorst, 2014). And in contrast to the CPT, the incorporation of social-evaluative threat in singing tasks means that they are likely to activate the HPA axis (found to be activated particularly in tasks with socialevaluative components).

In light of this growing body of evidence, we developed a novel stress induction protocol centered around singing, the Simple Singing Stress Procedure (SSSP). Importantly, we designed the SSSP with the purpose of improving currently available singing stressors. First, all of the singing stressors described in the previous paragraph involved arrangements with confederates, and some additionally involved long and complicated procedures. For example, the procedure introduced by Reschke-Hernández et al. (2017, described above) took around 15 min to complete and required two confederates to judge the singing performance and also to monitor for errors throughout 5 min of the participant performing mental subtraction aloud. Likewise, Brouwer and Hogervorst's (2014) procedure took around $11 \mathrm{~min}$ to complete and required two confederates to act as fake participants. By contrast, the SSSP procedure is quick (under $5 \mathrm{~min}$ ), simple, and can be easily implemented by a single experimenter. Second, a notable limitation of previous singing stress protocols is that they are generally geared towards eliciting (and measuring) stress in a single group of participants. That is, these singing protocols lack a validated no-stress control group with which to compare performance/physiological measures of the stress group, and instead only allow for inferences about the effect of the stressor relative to pre-stressor baseline (within-subjects). We designed the SSSP to include both a stress group and a nostress control group. Critically, comparing the stress group and no-stress group in our procedure allows for stronger inferences to be made about the causal influence of stress on variables of interest.

In the current study, we sought to investigate the efficacy of the SSSP as an alternative to existing stress induction protocols. We examined the differences between stress and nostress groups on HPA axis activation (measured by salivary cortisol), sympathetic nervous system activation (measured by blood pressure), and subjective ratings of stress. All materials and data can be accessed at https://osf.io/4573y.

\section{Method}

\section{Participants}

Eighty-three students ( 56 females; age $M=20.16$ years, SEM $=0.67$ years) from an undergraduate psychology course at UNSW Sydney participated in the study for course credit. All participants completed the study between the hours of 12:00 pm and 6:00 pm; natural cortisol levels are relatively stable during this period (Kirschbaum \& Hellhammer, 1994). All participants were instructed to refrain from intense physical exercise for the $4 \mathrm{~h}$ before the study, consuming caffeine or smoking $2 \mathrm{~h}$ before the study, and eating or drinking acidic drinks $1 \mathrm{~h}$ before the study, since these activities are known to influence levels of salivary cortisol (Kirschbaum, Klauer, Filipp, \& Hellhammer, 1995). Six participants were excluded from the study because they did not pass a screening questionnaire (see below). The remaining participants were assigned to the stress group or no-stress group in an alternating fashion based on order of arrival ( $n=38$ per group). The study was approved by the UNSW Human Research Ethics Advisory 
Panel (Psychology), and all participants provided informed consent to participate.

\section{Apparatus and materials}

Participants viewed stimuli on a 23-inch monitor. Questionnaires were presented via Inquisit, and the stressinduction procedure was presented using MATLAB with Psychophysics Toolbox extensions (Kleiner et al., 2007). Participants in the stress group were recorded during the singing task using a separate laptop with an inbuilt camera and microphone.

Kessler Psychological Distress Scale We used the Kessler Psychological Distress Scale (K10; Kessler et al. 2002) as a screening questionnaire for participants who may have been vulnerable to undue distress from the stress-induction procedure. This questionnaire asked participants about how often they had experienced symptoms of anxiety and depression in the previous 30 days. Participants responded to ten items using a five-point Likert scale that ranged from "none of the time" to "all of the time". Six participants scored 30 or above on the Kessler scale, and were not required to complete the stress procedure detailed below.

Simple Singing Stress Procedure (SSSP) We designed the SSSP to include an initial singing phase (Phase 1), followed by a later singing phase (Phase 2). We told participants that Phase 2 would be video- and audio-recorded to be later assessed by a panel of experimenters. Between these two phases, all participants completed a computer-based cognitive task (a visual search task, similar to that used by Pearson et al., 2016). We do not describe this cognitive task or its results in detail here, since it lies beyond the focus of the current article. We designed the SSSP to comprise two singing phases because we expected that the prospect of singing again would maintain stress at a high level throughout the intervening period (with the intention that a researcher interested in the causal impact of stress on a particular task could present that task during this intervening period). During each of Phases 1 and 2, those in the no-stress control group simply read aloud song lyrics. For all participants, this task was referred to as the 'song lyrics task'. For more details of the SSSP, see Procedure section.

Blood pressure measures Systolic and diastolic blood pressure (BP) were used to index levels of sympathetic nervous system activation (Schwabe, Tegenthoff, Höffken, \& Wolf, 2010). An Omron HEM7121 Blood Pressure Monitor was used to measure systolic and diastolic BP at four different time-points during the experiment (see Procedure). At each of these timepoints, the monitor's occluding cuff was placed around the participant's upper right arm and a measure taken by the experimenter.

Salivary cortisol measures Salivary cortisol was used as a measure of HPA axis activation (Kirschbaum \& Hellhammer, 1994). SalivaBio Oral Swabs (Salimetrics, State College, PA) were used to obtain saliva samples at three different time-points during the task (see Procedure). On each sampling occasion, participants placed a cotton swab under the tip of their tongue for $2 \mathrm{~min}$. Swabs were then placed in tubes and stored in a freezer $\left(-20^{\circ} \mathrm{C}\right)$. The samples were assayed for cortisol after all participants had been tested. Samples were centrifuged, and cortisol was measured using the Salimetrics cortisol immunoassay kit (Salimetrics, State College, PA). Due to a lack of oral swabs, the last five participants (two in the stress group, three in the no-stress group) did not provide saliva samples for cortisol analysis - these participants completed all other measures reported here.

Retrospective self-reported stress questionnaire At the end of the experiment, all participants retrospectively reported their levels of stress. First, to capture how effective the SSSP was in eliciting acute stress, we asked all participants "Looking back, how stressed did you feel after the FIRST song lyrics task?" ('Immediate' stress rating). Second, to capture participants' enduring levels of stress throughout the computer task, we also asked participants "Looking back, how stressed did you feel throughout the attention task, knowing that you would have to complete the song lyrics task again?" ('Throughout' stress rating). For each of these questions, participants responded on a visual analogue scale ranging from 0 ("Not at all stressed") to 100 ("Extremely stressed").

\section{Procedure}

Figure 1 shows an overview of the procedure. On arrival, participants first provided informed consent, and subsequently completed the Kessler screening questionnaire. Those who passed the screening questionnaire then provided a baseline measure of blood pressure (BP1) and a baseline salivary cortisol sample (Cort1).

Participants then completed Phase 1 of the SSSP. Participants in the stress condition were told: "Later on you will be asked to think of a song that you can sing to the experimenter. Your performance will be assessed for memory of the lyrics, in addition to the tone, pitch, and rhythm of your singing. You will be assigned a particular category and will have $60 \mathrm{~s}$ to prepare a song from that category. Please note that your performance will also be recorded. You now have the chance to practice singing a song (from a different category to that used later)". For this practice phase, participants were instructed to sing a song from the category 'love songs'. They were also told that for the later singing session, they 


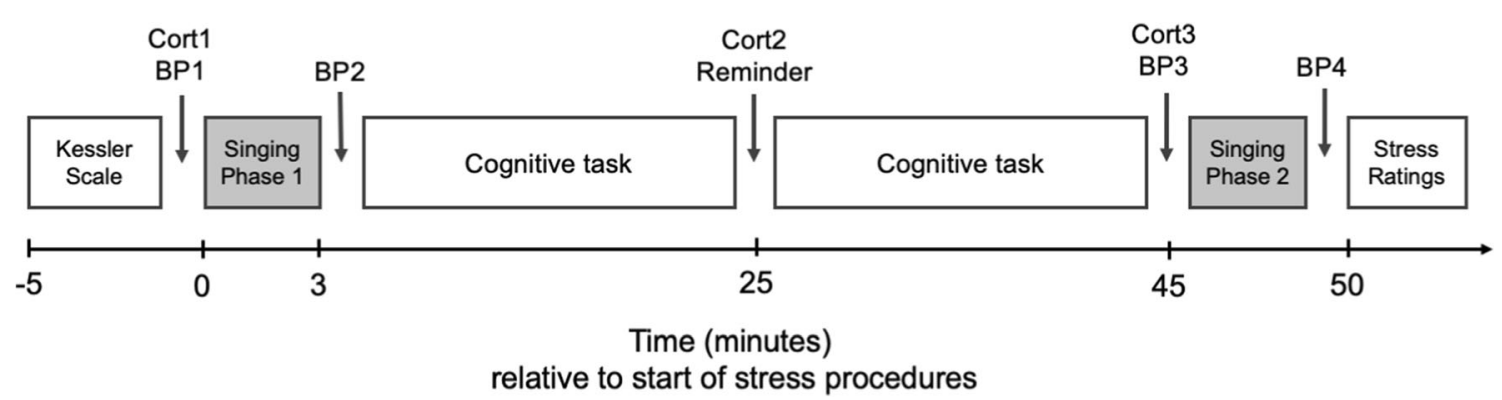

Fig. 1 A timeline of events for the study, including the stress procedures (Singing Phase 1 and Singing Phase 2), physiological measures, and questionnaires. Time 0 marks the start of the stress procedures. Blood pressure was measured four times during the experiment: immediately

would only be told which category to choose a song from immediately before they were required to sing. Our aim here was to discourage participants in the stress condition from attempting to remember and prepare a song for the assessment phase in advance, by ensuring they did not know the category of song they would have to sing later.

The experimenter started an on-screen, 60-s countdown and set up an audio- and video-recording program on a laptop that was positioned in front of the participant. If participants could not think of a song with $20 \mathrm{~s}$ remaining on the timer, the experimenter provided a list of popular love songs as recommendations. When the timer reached zero, the experimenter started the laptop recording; participants then sang their song of choice for about one verse, or until they could no longer remember the lyrics to the song. The experimenter remained in the room, standing behind the participant, while they sang. When the participant had finished singing, the recording was stopped. While the laptop was still positioned in front of the participant, the experimenter scanned through 3-4 points in the recording, telling participants that they needed to "check the recording had worked". This element of the procedure was used to increase the social evaluative nature of the task (similar to that used by Harris, 2001). Participants were then reminded that the assessment phase would be similar to the practice phase, except (1) the song category would be different, (2) they would actually be evaluated on their test phase performance, and (3) the experimenter would not give recommendations of songs.

By contrast, participants in the no-stress condition were not required to sing a song; instead, they were simply asked to read out song lyrics from a printed sheet. At the start of Phase 1, participants in the no-stress condition were told: "Later on, you will be asked to read out some song lyrics that appear on the screen. You will now have the chance to practice reading out song lyrics". The experimenter started the 60 -s countdown, and when it reached zero, participants read out three lines of lyrics from the song 'Lucy in the Sky with Diamonds' by The Beatles: "Lucy in the sky with diamonds/ Lucy in the sky with diamonds/ Lucy in the sky with diamonds". They before and after singing Phase 1 (BP1, BP2), and immediately before and after singing Phase 2 (BP3, BP4). Cortisol was measured three times during the experiment: immediately before Singing Phase 1 (Cort1), 25-30 min after (Cort2), and 40-45 min after (Cort3)

were not recorded, nor were they told that they would be assessed based on their performance. The requirement for participants in the no-stress condition to vocalize the written lyrics was designed to approximate the motor actions required in the stress condition, so that any differences in outcome measures could be attributed to the psychological difference between conditions rather than reflecting a difference in motor activity (see Brouwer \& Hogervorst, 2014).

After completing Phase 1 of the SSSP in their respective conditions, all participants had their blood pressure measured again (BP2). Since cortisol does not peak until 25 to $45 \mathrm{~min}$ after the start of an acute stressor (Goodman, Janson, \& Wolf, 2017), we did not take a second cortisol measurement at this time.

All participants were then set up to start on the cognitive (visual search) task. Around $25 \mathrm{~min}$ from the start of Phase 1, participants took a break from the visual search task and the experimenter collected the second cortisol measure to capture the start of the peak cortisol response (Cort2). All participants were then reminded that they would soon be required to complete Phase 2 of the SSSP. The aim of this reminder was to amplify levels of anticipatory stress in the stress group. Participants then took approximately 15-20 min to complete the rest of the visual search task. The experimenter then collected the final cortisol sample (Cort3), which would have occurred around 40-45 min after the onset of Phase 1, followed by a measure of blood pressure (BP3).

Participants then completed the Phase 2 of the SSSP in their respective conditions. Those in the stress condition were informed that they would now have to sing a song from the category 'Christmas songs'. They were also reminded that their performance in this phase would be later assessed by a panel of experimenters. Participants then proceeded as in the practice phase: after a 60 -s countdown, participants sang their song of choice, and the experimenter recorded audio and video on a laptop. Participants in the no-stress group were instead told that they would once again have to read song lyrics as they did in Phase 1. Here, these participants read aloud three lines from the song 'Diamonds' by Rihanna: "Shine bright 
like a diamond/ Shine bright like a diamond/ Shine bright like a diamond". After completing Phase 2, all participants had their blood pressure measured for the final time (BP4). Finally, participants completed the retrospective selfreported stress questionnaire before being debriefed about the study.

\section{Results}

\section{Cortisol response}

Figure 2a shows salivary cortisol levels across measurement occasions. These data were analyzed using a 2 (stress group: stress vs. no-stress) $\times 3$ (occasion: Cort1, Cort 2 , Cort 3 ) mixed ANOVA. There was a significant main effect of measurement occasion, $F(2,138)=25.87, p<.001, \eta_{\mathrm{p}}{ }^{2}=.273$, but no significant main effect of stress group, $F(1,69)=1.25, p=$ $.27, \eta_{\mathrm{p}}{ }^{2}=.018$. Notably, there was a significant interaction between stress group and measurement occasion, $F(2,138)=$ $14.06, p<.001, \eta_{\mathrm{p}}{ }^{2}=.169$, with an increase in cortisol in the stress group relative to the no-stress group across measurement occasions. Pre-planned, Bonferroni-corrected analyses of simple effects examined the one-tailed hypothesis that salivary cortisol would be higher in the stress group than the nostress group at Cort 2 and Cort3 (we would not expect a difference at Cort1 as this was prior to the stress-induction procedure). These analyses revealed that salivary cortisol levels did not differ significantly between stress and no-stress groups at Cort2 (around $25 \mathrm{~min}$ after the onset of singing Phase 1), $t(69)=.50$, Bonferroni-corrected $p\left(p_{b}\right)=.62, d=.12$. However, the stress group exhibited significantly higher cortisol levels than the no-stress group at Cort3, $t(69)=2.12, p_{\boldsymbol{b}}=$ $.037, d=.50$, around 45 min after the onset of singing Phase 1 , which is consistent with previous estimates of the latency of the peak salivary cortisol stress response (Goodman, Janson, \& Wolf, 2017).

\section{Retrospective self-reported stress ratings}

The stress group reported feeling significantly more stressed than the no-stress group immediately after the initial stressor $($ singing Phase 1$), t(74)=7.58, p<.001, d=1.74$, and throughout the subsequent visual search task, $t(74)=5.11, p$ $<.001, d=1.17$ (Fig. 2b).

\section{Blood pressure}

Systolic and diastolic blood pressure values were recorded on each occasion. We examined changes in each blood pressure measurement using separate 2 (stress group: stress vs. nostress) $\times 4$ (occasion: BP1, BP2, BP3, BP4) mixed ANOVAs.

Systolic blood pressure Figure 2c shows systolic blood pressure across measurement occasions, in each group. We found a significant main effect of measurement occasion, $F(3,222)=$ a

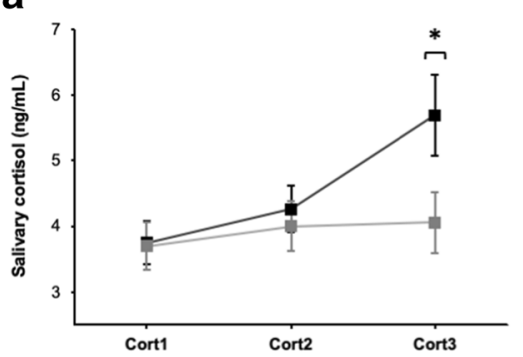

C

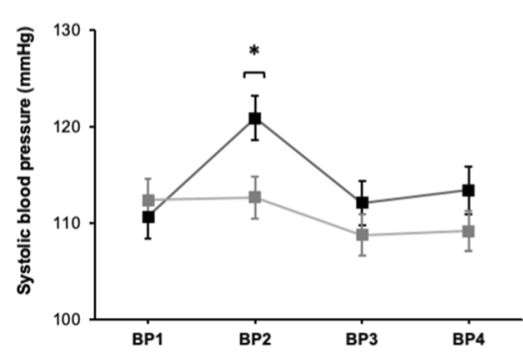

b

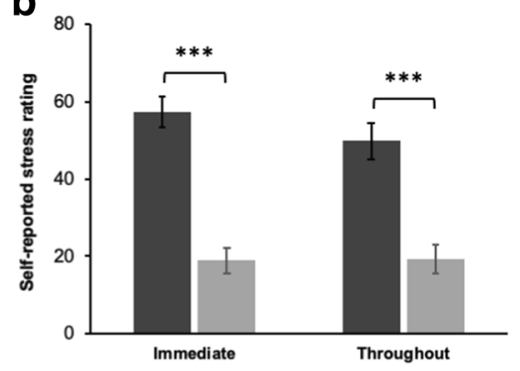

d

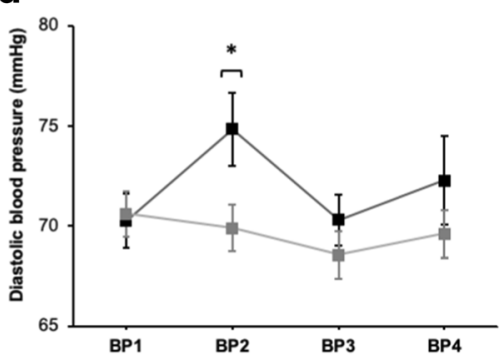

Fig. 2 a Mean salivary cortisol levels at each of the measurement occasions: immediately before Phase 1 (Cort1), 25-30 min after (Cort2), and 40-45 min after (Cort3). b Mean retrospective selfreported ratings of stress experienced immediately after Phase 1 (Immediate) and throughout the subsequent cognitive task (Throughout). c Mean systolic blood pressure in the stress and no-stress

groups at each of the measurement occasions: BP1 and BP2 were recorded immediately before and after Phase 1, and BP3 and BP4 were recorded immediately before and after Phase 2. d Mean diastolic blood pressure in the stress and no-stress groups at each of the measurement occasions. Error bars represent SEM. $* p<.05, * * * p<.001$ 
$15.55, p<.001, \eta_{\mathrm{p}}{ }^{2}=.17$, while the main effect of group was not significant $F(1,74)=1.50, p=.224, \eta_{\mathrm{p}}{ }^{2}=.02$. More importantly, we found a significant interaction between stress group and measurement occasion, $F(3,222)=7.79, p<.001$, $\eta_{\mathrm{p}}{ }^{2}=.10$. Pre-planned, Bonferroni-corrected analyses of simple effects examined the one-tailed hypothesis that systolic blood pressure would be higher in the stress group than the no-stress group at timepoints BP2, BP3, and BP4 (we would not expect a difference at BP1 as this was prior to the stressinduction procedure). These analyses revealed that the stress group exhibited a significantly higher mean systolic blood pressure than the no-stress group at BP2 (immediately after the SSSP practice phase), $t(74)=2.64, p_{b}=.015, d=.61$. The two groups did not significantly differ at BP3, $t(74)=1.07, p_{b}$ $=.433, d=.25$, nor BP4, $t(74)=1.33, p_{b}=.281, d=.31$ (immediately before and after the SSSP assessment phase, respectively).

Diastolic blood pressure We found a significant main effect of measurement occasion, $F(3,222)=3.02, p=.031, \eta_{\mathrm{p}}{ }^{2}=.04$, while the main effect of group was not significant $F(1,74)=$ $1.80, p=.18, \eta_{\mathrm{p}}{ }^{2}=.02$ (Fig. 2d). The interaction between group and occasion approached significance, $F(3,222)=$ 2.43, $p=.066, \eta_{\mathrm{p}}{ }^{2}=.032$. Pre-planned, Bonferronicorrected analyses were used to examine the one-tailed hypothesis that diastolic blood pressure would be higher in the stress group than the no-stress group at timepoints BP2, BP3 and BP4. These analyses revealed that the stress group exhibited a significantly higher mean diastolic blood pressure than the no-stress group at BP2, $t(74)=2.284, p_{b}=.038, d=.52$. The two groups did not significantly differ at BP3, $t(74)=$ $1.022, p_{b}=.47, d=.23$, nor BP4, $t(74)=1.057, p_{b}=.441, d$ $=24$. While the interaction between group and measurement occasion did not reach significance when assessed over all four measurement occasions, if analysis was restricted to occasions BP1 and BP2 (immediately before and after the first phase of the stress-induction procedure), the group $\times$ occasion interaction was significant for diastolic pressure, $F(1,74)=$ $10.1, p=.002, \eta_{\mathrm{p}}{ }^{2}=.120$. Thus the stress induction manipulation did induce a significant increase in diastolic blood pressure. Presumably, the non-significant interaction in the overall analysis reflects an influence of noise in the data from later time-points at which blood pressure was more similar across groups.

\section{Discussion}

The aim of the current study was to validate the use of the Simple Singing Stress Procedure (SSSP) as an alternative to existing stress protocols. Our procedure proved to be successful in inducing acute stress. We found significantly elevated blood pressure in participants in the stress condition relative to the no-stress condition, in the period immediately after Phase 1. Participants in the stress condition also reported experiencing significantly greater subjective levels of stress immediately after Phase 1 , and this increased level of stress was maintained over $\sim 45 \mathrm{~min}$ following the initial stressor, in anticipation of Phase 2. Finally, our protocol produced significantly higher salivary cortisol levels in participants in the stress condition, relative to participants in the no-stress condition.

One question that remains unclear from the current study is whether the between-group differences in physiological markers of stress (salivary cortisol and blood pressure) were driven by an acute stress response from Phase 1, anticipatory stress towards Phase 2, or a combination of both these influences. This question is particularly pertinent to group differences at Cort3 (immediately prior to the second singing phase): given the delay in salivary cortisol changes following HPA axis activation (Goodman et al. 2017), this measurement reflects stress experienced around the time of Phase 1. However, it is not clear whether Cort3 more specifically captures group differences in HPA axis activation during Phase 1 (which would reflect an acute stress response) or the short period after Phase 1 (which would reflect ongoing anticipatory stress). Moreover, we did not collect another salivary cortisol response after Cort3. Collecting another sample approximately 20 min after Cort 3 would have provided a measure of HPA axis activation relating to the period in the middle of the cognitive task - that is, the period in which participants' selfreport ratings revealed anticipatory stress about having to sing again. Future studies could investigate this issue further in order to clarify the dissociable roles of acute and anticipatory stress in the SSSP on HPA axis function.

Finally, one notable finding was the lack of significant differences between groups in blood pressure on occasion BP4, immediately after the second singing phase. This finding may suggest that participants were no longer stressed after the second singing session - perhaps because it was predictable in time (and hence they were prepared for it), or because they experienced a sense of relief following the completion of the stress procedures. Nevertheless, this finding (as well as the ambiguity of the role of anticipatory stress on salivary cortisol) does not negate the finding that participants reported being stressed throughout the computer task. Hence, we would recommend that researchers using the SSSP keep tasks of interest between the two singing phases, as stress levels may drop rapidly following the second singing session.

\section{The SSSP versus existing stress-induction procedures}

The SSSP has important advantages over existing, commonly used stress protocols. Our procedure is much simpler than the current 'gold standard' Trier Social Stress Test (TSST): it can be efficiently and quickly implemented by a single experimenter without the requirement for confederates, with the time 
of singing sessions totaling just $5 \mathrm{~min}$ (as opposed to around $20 \mathrm{~min}$ for the TSST). In contrast to the alternative Cold Pressor Test (CPT) protocol, the SSSP incorporates an element of social-evaluative stress, and hence may be more likely to generate experiences that are subjectively felt as 'stress' (rather than pain in the CPT). Indeed, whereas increases in subjective ratings of stress produced by the CPT are typically relatively modest (e.g., effect sizes of $d=0.23$ to 0.46 reported by Brady et al. 2006) and short-lived (with ratings returning to baseline within 5 min: McRae et al. 2006), our procedure generated substantial increases in subjective ratings of stress ('Immediate' ratings, effect size $d=1.74$ ) that persisted for at least $45 \mathrm{~min}$ ('Throughout' ratings, effect size $d=1.17$ ).

As noted earlier, we are not the first to suggest the use of singing as a time-efficient and social-evaluative stress-induction procedure. However, previous protocols based on singing (e.g., Brouwer \& Hogervorst, 2014; Harris, 2001; ReschkeHernández et al. 2017) are limited by their requirement for confederates, and by the lack of validated no-stress control groups. This latter point is particularly important: researchers investigating the causal influence of stress on a particular task will typically want to compare performance of that task under stressed conditions with performance under unstressed conditions that are otherwise similar. For this reason, we designed the SSSP to include both a stress group and a no-stress group, allowing for experimental manipulations of stress. In the current study, the experimental manipulation of stress allows us to make strong inferences about the specific factors driving our findings. First, we demonstrated that both systolic and diastolic blood pressure were significantly higher in the stress group relative to the no-stress group, immediately after Phase 1 of the SSSP. These findings suggest that the specific experience of singing leads to the activation of the sympathetic nervous system, as opposed to non-specific factors, such as the countdown, being in the presence of the experimenter, vocalizing, or having blood pressure measured (which the no-stress controls also experienced). Similarly, we found higher levels of salivary cortisol in the stress group relative to the no-stress group, suggesting that singing in the SSSP leads to activation of the HPA axis. This finding is in line with previous work that suggests social-evaluative elements of laboratory stressors are primarily responsible for HPA axis activation (Dickerson \& Kemeny, 2004). In our stress procedure, participants were required to sing aloud in front of the experimenter, and they were aware that their performance was being recorded. This procedure effectively placed our participants in a situation of heightened social threat and increased the potential for negative evaluation. It is likely that this social-evaluative context was the key precipitator of HPA axis activation, which is widely considered to be crucial to demonstrating the validity of laboratory stress procedures (Goodman, Janson, \& Wolf, 2017; Schwabe, Haddad, \& Schachinger, 2008). Overall, the SSSP is notable among singing-based stress protocols in that it provides experimenters with the ability to make inferences about the causal role of the singing stressor on variables of interest.

The SSSP induced an increase in salivary cortisol from baseline of around $1.96 \mathrm{ng} / \mathrm{ml}(5.38 \mathrm{nmol} / \mathrm{l})$, with a large effect size for the difference in change in cortisol levels over time between the stress and no-stress groups $\left(\eta_{\mathrm{p}}{ }^{2}=.169\right.$, which corresponds to $d \approx 0.90$ ). It is difficult to compare the size of this cortisol response with that elicited by existing stress-induction procedures such as the TSST, CPT and Socially Evaluated CPT (SECPT) given the earlier-noted heterogeneity in effect sizes reported in the literature. Considering instead individual studies that have systematically compared effects of different stressors on cortisol levels (Giles, Mahoney, Brunyé, Taylor, \& Kanarek, 2014; Schwabe, Haddad, \& Schachinger, 2008; Skoluda et al., 2015), it would seem that the cortisol effect induced by the current SSSP is likely to be on a par with that generated by the TSST and SECPT (around 4-6 nmol/l), and greater than that produced by the standard CPT (around $2 \mathrm{nmol} / \mathrm{l}$ ). However, given the wide variance in responses observed across different studies, a systematic, within-study contrast of cortisol response elicited by the SSSP versus other stress-induction procedures would be a useful target for future research.

The SSSP bears further comparison with the SECPT (Schwabe, Haddad, \& Schachinger, 2008). Both stressinduction procedures: (1) are brief ( $<5 \mathrm{~min})$; (2) can be implemented by a single experimenter; (3) incorporate an element of social evaluation and elicit activation of the HPA axis; and (4) have a standardized control group (no-stress 'reading' group for the SSSP; immersion of the hand in lukewarm water for the SECPT) allowing for the investigation of the causal influence of stress. As discussed earlier, the SECPT is limited by practical considerations for the purchasing, storage, preparation, and disposal of ice for the ice-water bath. By contrast, the SSSP does not involve costs and is simple to administer.

\section{Remaining questions}

It seems plausible that there are individual differences in the extent to which the requirement to sing aloud to an experimenter is stress-inducing: while for most people this experience is likely to be highly stressful, some participants (particularly those with experience of singing in public) may be less affected. We did not assess participants' singing background in the current study, and hence could not test its relationship with stress experienced from the SSSP. Future research could additionally assess singing experience to examine this issue, and/or include singing experience as a covariate to increase power in statistical analyses.

It is also worth noting that the current study examined changes in blood pressure as a measure of sympathetic nervous system activation, and we found that the stress condition 
of the SSSP produced significant increases in both systolic and diastolic pressure relative to the no-stress condition. While blood pressure has previously been used as a cardiovascular index of sympathetic nervous system activation in studies using stress-induction procedures (Andrews \& Pruessner, 2013; Childs, Dlugos, \& De Wit, 2010; Jezova et al. 2004; Schwabe \& Schächinger, 2018), other studies have instead measured heart rate (Brouwer \& Hogervorst, 2014; Kirschbaum et al. 1993; von Dawans, Kirschbaum, \& Heinrichs, 2011; Schwabe, Haddad, \& Schachinger, 2008). Future studies could examine the influence of the SSSP on heart rate as an alternative cardiovascular measure, which would corroborate and extend the current findings with regard to effects on blood pressure.

\section{Conclusions}

Here, we have demonstrated that the SSSP is an efficacious and valid alternative to existing laboratory stress procedures. At its core, the SSSP is a simple procedure that capitalizes on the inherently stressful and social-evaluative nature of singing in front of another person, and can be easily implemented by a single experimenter. Hence, it can be used to overcome the practical challenges and theoretical limitations of commonly used stress procedures such as the Trier Social Stress Test and the Cold Pressor Test. Notably, the SSSP is the first singing stress procedure that allows for experimental manipulations of stress, given the inclusion of a no-stress control group that has here been validated. Critically, such a design might be particularly useful for experimenters who wish to use a stress procedure to examine the causal influence of stress on various aspects of health and cognition.

Author Note This research was funded by Australian Research Council grants DP170101715 and DP200101314. Poppy Watson was supported by an Australian Research Council Discovery Early Career Researcher Award (DE200100591).

\section{References}

al'Absi, M., Petersen, K. L., \& Wittmers, L. E. (2002). Adrenocortical and hemodynamic predictors of pain perception in men and women. Pain, 96(1), 197-204.

Allen, A. P., Kennedy, P. J., Cryan, J. F., Dinan, T. G., \& Clarke, G. (2014). Biological and psychological markers of stress in humans: Focus on the Trier Social Stress Test. Neuroscience \& Biobehavioral Reviews, 38(1), 94-124.

Allen, A. P., Kennedy, P. J., Dockray, S., Cryan, J. F., Dinan, T. G., \& Clarke, G. (2017). The Trier Social Stress Test: Principles and practice. Neurobiology of Stress, 6, 113-126.

Andrews, J., \& Pruessner, J. C. (2013). The combined propranolol/TSST paradigm: A new method for psychoneuroendocrinology. PLoS One, 8(2), e57567.

Bloom, L. J., and Trautt, G. M. (1977). Finger pulse volume as a measure of anxiety: Further evaluation. Psychophysiology 14(6), 541-544.
Brady, K. T., Back, S. E., Waldrop, A. E., McRae, A. L., Anton, R. F., Upadhyaya, H. P., ... \& Randall, P. K. (2006). Cold pressor task reactivity: Predictors of alcohol use among alcohol-dependent individuals with and without comorbid posttraumatic stress disorder. Alcoholism: Clinical and Experimental Research, 30(6), 938-946.

Brouwer, A. M., \& Hogervorst, M. A. (2014). A new paradigm to induce mental stress: The Sing-a-Song Stress Test (SSST). Frontiers in Neuroscience, 8(1), 224.

Bryant, R. A. (2011). Acute stress disorder as a predictor of posttraumatic stress disorder: A systematic review. The Journal of Clinical Psychiatry. 72(2), 233-239.

Buchanan, T. W., Tranel, D., \& Adolphs, R. (2006). Impaired memory retrieval correlates with individual differences in cortisol response but not autonomic response. Learning \& Memory, 13(3), 382-387.

Bullinger, M., Naber, D., Pickar, D., Cohen, R. M., Kalin, N. H., Pert, A., \& Bunney Jr, W. E. (1984). Endocrine effects of the cold pressor test: Relationships to subjective pain appraisal and coping. Psychiatry Research, 12(3), 227-233.

Caspi, A., Sugden, K., Moffitt, T. E., Taylor, A., Craig, I. W., Harrington, H., ... \& Poulton, R. (2003). Influence of life stress on depression: Moderation by a polymorphism in the 5-HTT gene. Science, 301(5631), 386-389.

Childs, E., Dlugos, A., \& De Wit, H. (2010). Cardiovascular, hormonal, and emotional responses to the TSST in relation to sex and menstrual cycle phase. Psychophysiology, 47(3), 550-559.

Cornelisse, S., van Stegeren, A. H., \& Joëls, M. (2011). Implications of psychosocial stress on memory formation in a typical male versus female student sample. Psychoneuroendocrinology, 36(4), 569578.

Dickerson, S. S., \& Kemeny, M. E. (2004). Acute stressors and cortisol responses: A theoretical integration and synthesis of laboratory research. Psychological Bulletin, 130(3), 355.

Duncko, R., Cornwell, B., Cui, L., Merikangas, K. R., \& Grillon, C. (2007). Acute exposure to stress improves performance in trace eyeblink conditioning and spatial learning tasks in healthy men. Learning \& Memory, 14(5), 329-335.

Eisenberg, N., Fabes, R. A., Bustamante, D., Mathy, R. M., Miller, P. A., \& Lindholm, E. (1988). Differentiation of vicariously induced emotional reactions in children. Developmental Psychology, 24(2), 237245.

Ekman, P., Levenson, R. W., \& Friesen, W. V. (1983). Autonomic nervous system activity distinguishes among emotions. Science, 221(4616), 1208-1210.

Esterman, M., DeGutis, J., Mercado, R., Rosenblatt, A., Vasterling, J. J., Milberg, W., \& McGlinchey, R. (2013). Stress-related psychological symptoms are associated with increased attentional capture by visually salient distractors. Journal of the International Neuropsychological Society, 19(7), 835-840.

Estryn-Behar, M., Kaminski, M., Peigne, E., Bonnet, N., Vaichere, E., Gozlan, C., ... \& Giorgi, M. (1990). Stress at work and mental health status among female hospital workers. Occupational and Environmental Medicine, 47(1), 20-28.

Geisser, M. E., Robinson, M. E., \& Pickren, W. E. (1992). Differences in cognitive coping strategies among pain-sensitive and pain-tolerant individuals on the cold-pressor test. Behavior Therapy, 23(1), 3141.

Giles, G. E., Mahoney, C. R., Brunyé, T. T., Taylor, H. A., \& Kanarek, R. B. (2014). Stress effects on mood, HPA axis, and autonomic response: Comparison of three psychosocial stress paradigms. PloS One, 9(12), e113618.

Gluck, M. E., Geliebter, A., Hung, J., \& Yahav, E. (2004). Cortisol, hunger, and desire to binge eat following a cold stress test in obese women with binge eating disorder. Psychosomatic Medicine, 66(6), 876-881.

Goodman, W. K., Janson, J., \& Wolf, J. M. (2017). Meta-analytical assessment of the effects of protocol variations on cortisol responses 
to the Trier Social Stress Test. Psychoneuroendocrinology, 80(1), 26-35.

Harris, C. R. (2001). Cardiovascular responses of embarrassment and effects of emotional suppression in a social setting. Journal of Personality and Social Psychology, 81(5), 886-897.

Hines, E.A. \& Brown, G.E. (1932). A standard stimulus for measuring vasomotor reactions: Its application in the study of hypertension. Proc. Staff Meet Mayo Clin. 7, 332.

Jezova, D., Makatsori, A., Duncko, R., Moncek, F., \& Jakubek, M. (2004). High trait anxiety in healthy subjects is associated with low neuroendocrine activity during psychosocial stress. Progress in Neuro-Psychopharmacology and Biological Psychiatry, 28(8), $1331-1336$.

Kalia, M. (2002). Assessing the economic impact of stress: The modernday hidden epidemic. Metabolism: Clinical and Experimental, 51(6), 49-53.

Kessler, R. C., Andrews, G., Colpe, L. J., Hiripi, E., Mroczek, D. K., Normand, S. L., ... \& Zaslavsky, A. M. (2002). Short screening scales to monitor population prevalences and trends in nonspecific psychological distress. Psychological Medicine, 32(6), 959-976.

Kim, P., Evans, G. W., Angstadt, M., Ho, S. S., Sripada, C. S., Swain, J. E., ... \& Phan, K. L. (2013). Effects of childhood poverty and chronic stress on emotion regulatory brain function in adulthood. Proceedings of the National Academy of Sciences, 110(46), 18442-18447.

King, S., \& Laplante, D. P. (2005). The effects of prenatal maternal stress on children's cognitive development: Project Ice Storm. Stress, 8(1), 35-45.

Kirschbaum, C., \& Hellhammer, D. H. (1994). Salivary cortisol in psychoneuroendocrine research: Recent developments and applications. Psychoneuroendocrinology, 19(4), 313-333.

Kirschbaum, C., Klauer, T., Filipp, S. H., \& Hellhammer, D. H. (1995). Sex-specific effects of social support on cortisol and subjective responses to acute psychological stress. Psychosomatic Medicine, 57(1), 23-31.

Kirschbaum, C., Pirke, K. M., \& Hellhammer, D. H. (1993). The 'Trier Social Stress Test': A tool for investigating psychobiological stress responses in a laboratory setting. Neuropsychobiology, 28(1), 7681.

Kleiner, M., Brainard, D.H., \& Pelli, D., Ingling, A., Murray, R., \& Broussard, C. (2007). What's new in Psychtoolbox-3? Perception, 36(14), ECVP Abstract Supplement.

Kowalczyk, W. J., Evans, S. M., Bisaga, A. M., Sullivan, M. A., \& Comer, S. D. (2006). Sex differences and hormonal influences on response to cold pressor pain in humans. The Journal of Pain, 7(3), $151-160$.

Kuhlmann, S., Piel, M., \& Wolf, O. T. (2005). Impaired memory retrieval after psychosocial stress in healthy young men. Journal of Neuroscience, 25(11), 2977-2982.

Lupien, S. J., Fiocco, A., Wan, N., Maheu, F., Lord, C., Schramek, T., \& Tu, M. T. (2005). Stress hormones and human memory function across the lifespan. Psychoneuroendocrinology, 30(3), 225-242.

Mantsch, J. R., Baker, D. A., Funk, D., Le, A. D., \& Shaham, Y. (2016). Stress-induced reinstatement of drug seeking: 20 years of progress. Neuropsychopharmacology, 41(1), 335-356.

Mather, M., \& Lighthall, N. R. (2012). Risk and reward are processed differently in decisions made under stress. Current Directions in Psychological Science, 21(1), 36-41.

McCullough, A. M., Ritchey, M., Ranganath, C., \& Yonelinas, A. (2015). Differential effects of stress-induced cortisol responses on recollection and familiarity-based recognition memory. Neurobiology of Learning and Memory, 123, 1-10.

McRae, A. L., Saladin, M. E., Brady, K. T., Upadhyaya, H., Back, S. E., \& Timmerman, M. A. (2006). Stress reactivity: Biological and subjective responses to the cold pressor and Trier social stressors.
Human Psychopharmacology: Clinical and Experimental, 21(6), 377-385.

Noble, K. G., Houston, S. M., Brito, N. H., Bartsch, H., Kan, E., Kuperman, J. M., .. \& \& Schork, N. J. (2015). Family income, parental education and brain structure in children and adolescents. Nature Neuroscience, 18(5), 773-778.

Payne, J., Jackson, E., Ryan, L., Hoscheidt, S., Jacobs, J., \& Nadel, L. (2006). The impact of stress on neutral and emotional aspects of episodic memory. Memory, 14(1), 1-16.

Pearson, D., Osborn, R., Whitford, T.J., Failing, M., Theeuwes, J., \& Le Pelley, M.E. (2016). Value-modulated oculomotor capture by taskirrelevant stimuli is a consequence of early competition on the saccade map. Attention, Perception, \& Psychophysics, 78, 2226-2240.

Reschke-Hernández, A. E., Okerstrom, K. L., Bowles Edwards, A., \& Tranel, D. (2017). Sex and stress: Men and women show different cortisol responses to psychological stress induced by the Trier Social Stress Test and the Iowa Singing Social Stress Test. Journal of Neuroscience Research, 95(1-2), 106-114.

Sänger, J., Bechtold, L., Schoofs, D., Blaszkewicz, M., \& Wascher, E. (2014). The influence of acute stress on attention mechanisms and its electrophysiological correlates. Frontiers in Behavioral Neuroscience, $8,353$.

Schoofs, D., Wolf, O. T., \& Smeets, T. (2009). Cold pressor stress impairs performance on working memory tasks requiring executive functions in healthy young men. Behavioral Neuroscience, 123(5), $1066-1075$.

Schwabe, L., Haddad, L., \& Schachinger, H. (2008). HPA axis activation by a socially evaluated cold-pressor test. Psychoneuroendocrinology, 33(6), 890-895.

Schwabe, L., \& Schächinger, H. (2018). Ten years of research with the Socially Evaluated Cold Pressor Test: Data from the past and guidelines for the future. Psychoneuroendocrinology, 92, 155-161.

Schwabe, L., Tegenthoff, M., Höffken, O., \& Wolf, O. T. (2010). Concurrent glucocorticoid and noradrenergic activity shifts instrumental behavior from goal-directed to habitual control. Journal of Neuroscience, 30(24), 8190-8196.

Shields, G. S., Dunn, T. M., Trainor, B. C., \& Yonelinas, A. P. (2019). Determining the biological associates of acute cold pressor postencoding stress effects on human memory: The role of salivary interleukin-1ß. Brain, Behavior, and Immunity, 81, 178-187.

Shields, G. S., Sazma, M. A., McCullough, A. M., \& Yonelinas, A. P. (2017). The effects of acute stress on episodic memory: A metaanalysis and integrative review. Psychological bulletin, 143(6), 636-675.

Skoluda, N., Strahler, J., Schlotz, W., Niederberger, L., Marques, S., Fischer, S., ... \& Nater, U. M. (2015). Intra-individual psychological and physiological responses to acute laboratory stressors of different intensity. Psychoneuroendocrinology, 51, 227-236.

Smeets, T., Cornelisse, S., Quaedflieg, C. W., Meyer, T., Jelicic, M., \& Merckelbach, H. (2012). Introducing the Maastricht Acute Stress Test (MAST): A quick and non-invasive approach to elicit robust autonomic and glucocorticoid stress responses. Psychoneuroendocrinology, 37(12), 1998-2008.

Starcke, K., \& Brand, M. (2012). Decision making under stress: A selective review. Neuroscience and Biobehavioral Reviews, 36(4), 12281248.

Von Dawans, B., Kirschbaum, C., \& Heinrichs, M. (2011). The Trier Social Stress Test for Groups (TSST-G): A new research tool for controlled simultaneous social stress exposure in a group format. Psychoneuroendocrinology, 36(4), 514-522.

Wolf, O. T. (2009). Stress and memory in humans: Twelve years of progress?. Brain Research, 1293, 142-154.

Publisher's note Springer Nature remains neutral with regard to jurisdictional claims in published maps and institutional affiliations. 